\title{
Lag-Dependent Regularization for MLPs Applied to Financial Time Series Forecasting Tasks
}

\author{
Andrew Skabar \\ Department of Computer Science and Computer Engineering \\ La Trobe University, Victoria 3086 Australia \\ a.skabar@latrobe.edu.au
}

\begin{abstract}
The application of multilayer perceptrons to forecasting the future value of some time series based on past (or lagged) values of the time series usually requires very careful selection of the number of lags to be used as inputs, and this must usually be determined empirically. This paper proposes a regularization technique by which the influence that a lag has in determining the forecast value decreases exponentially with the lag, and is consistent with the intuitive notion that recent values should have more influence than less recent values in predicting future values. This means that in principle an infinite number of dimensions could be used. Empirical results show that the regularization technique yields superior performance on out-of-sample data compared with approaches that use a fixed number of inputs without lag-dependent regularization.
\end{abstract}

Keywords: Multilayer perceptrons, financial time series forecasting, weight regularization.

\section{Introduction}

Over the last two or so decades there has been much interest in applying multilayer perceptron (MLP) models to financial time series forecasting tasks. The appeal of MLPs is their property of being universal function approximators; that is, they are able to approximate any target function to arbitrary degree of accuracy [1]. However, the ability of a model to achieve good performance on in-sample data does not guarantee that it will perform well in forecasting out-of-sample data, and this is particularly a concern in the case of models such as MLPs, which, due to their complexity, together with the high level of noise present in financial time series, can very easily overfit the training data. It is not surprising, then, that there has been considerable debate about whether non-linear models such as MLPs are able to provide any better performance financial time series forecasting than linear or random walk models [2-4].

The main factor determining the complexity of an MLP model (and hence its propensity to overfit training data) is the number of weights, and this depends on two factors: the number of inputs to the model, and the number of hidden layer units. Since each input fans out to each hidden unit, the total number of weights will be a function of the product $D \cdot h$, where $D$ is the input dimensionality and $h$ is the number of hidden units; hence, choosing an appropriate value for these two parameters plays 
an important role in determining the overall complexity of the model. Fortunately, other means also exist for mitigating against the effect of model complexity. For example, it is common to include a regularization term into the error function, the purpose of which is to impose a penalty against large magnitude weights, thereby controlling the effective complexity of the model [5].

The main problem with which we are concerned in this paper is how to select an appropriate number of inputs. As is common practice on financial time series forecasting tasks, we assume that the input to the MLP is a vector of delayed returns $\left(r_{t-1}\right.$, $\left.r_{t-2}, \ldots, r_{t-D}\right)^{\mathrm{T}}$, where $r_{t-n}$ is the return $n$ days prior to day $t$ (and which we will refer to as the $n^{\text {th }}$ 'lag'). The problem, then, is how to select an appropriate value for $D$. If $D$ is too small, then we may miss out on detecting important patterns in the time series; if $D$ is too large then we run the risk of modeling noise in the training data, thereby leading to overfitting. A common practice in determining the optimum input dimensionality is to experiment with a range of values, and to then use some model selection criteria such as the Akaike Information Criterion (AIC) [6] or the Schwarz criterion (BIC) [7] to make the final selection [8]. Typically, the number of lags selected lies in the range two to five; however, there can be dramatic differences in performance observed by increasing the dimensionality by just one.

Motivated by the intuitive notion that recent values should have more influence than less recent values in predicting future values, in this paper we propose a weight regularization technique by which the influence that a lag has in determining the forecast value decreases exponentially with the lag. This means that in principle an infinite number of dimensions can be used. Empirical results show that the regularization technique yields superior performance on out-of-sample data compared with approaches that use a fixed number of inputs without lag-dependent regularization.

The remainder of this paper is structured as follows. In Section 2, we describe previous work, in which we used a generative model to predict the probability of an upward/downward direction of change in the value of a return series. Specifically, we show how the covariance matrix used to estimate densities can be parameterized in such a way that the influence of past returns decreases exponentially with time, thus allowing an effectively infinite input dimensionality. In Section 3 we show how this idea of exponentially decreasing influence of past returns can be implemented in discriminative models such as MLPs. Section 4 provides empirical results of applying the technique to several datasets, and Section 5 concludes the paper.

\section{Related Work}

In [9] we showed how the intuitive notion that recent values of the time series should to be more influential than less recent values could be implemented within a generative model. Assuming a set of examples $\mathbf{X}=\left\{\mathbf{x}_{1}, \mathbf{x}_{2}, \ldots, \mathbf{x}_{N}\right\}$ where $\mathbf{x}_{n}=\left(r_{n-1}, r_{n-2}, \ldots\right.$, $\left.r_{n-D}\right)^{T}$ is a vector of delayed returns on day $n$, the objective was to predict the posterior probability of a positive return on day $n$. Since each $\mathbf{x}$ belongs to one of two classes, which we will denote as $C_{+}$for positive returns and $C_{-}$for negative returns, the problem is thus a binary classification problem. Using a Parzen density estimation $[10,11]$, the probability density function for examples belonging to $C_{+}$can be estimated as 


$$
p\left(\mathbf{x} \mid C_{+}, \Sigma\right)=\frac{1}{\left|\mathbf{X}_{+}\right|} \frac{1}{(2 \pi)^{D / 2}\left|\sum\right|^{1 / 2}} \sum_{n=1}^{\left|\mathbf{X}_{+}\right|} \exp \left(-\frac{1}{2}\left(\mathbf{x}-\mathbf{x}_{+n}\right)^{T} \Sigma^{-1}\left(\mathbf{x}-\mathbf{x}_{+n}\right)\right),
$$

where $\sum$ is the covariance matrix and $\mathbf{X}_{+}$is the set of examples belonging to $C_{+}$. The density $p\left(\mathbf{x} \mid C_{-}, \Sigma\right)$ can be calculated similarly. These densities can then be combined using Bayes' Theorem to estimate the posterior probabilities $P\left(C_{+} \mid \mathbf{x}, \Sigma\right)$ and $P\left(C_{-} \mid \mathbf{x}, \Sigma\right)$, which represent the probability respectively of a positive and negative return.

Since we expect that the importance of lags to decrease with the length of the lag, this suggests that the kernel used to estimate a density should not be symmetrical (i.e., spherical), but of a form such that its width along dimensions corresponding to recent returns is smaller than its width along dimensions corresponding to less recent returns. Assuming that significance decreases exponentially with the length of the lag, we proposed the following expression for the variance in the direction corresponding to the $n^{\text {th }}$ lag:

$$
v_{t-n}=a \mathrm{e}^{k(n-1)}
$$

where $v_{t-n}(n \in\{1,2, \ldots, D\})$ is the variance of the kernel in the direction parallel to the axis corresponding to lag $r_{t-n}, k$ is an exponential scaling factor, and $a$ is the variance parallel to the first lag. These values are thus the diagonal components of the covariance matrix, with all off-diagonal components being equal to zero.

In general, the higher the variance of the time series, the larger will be the value of $a$. The value of $k$ will depend on how rapidly the influence of lags in predicting future values diminishes with the length of the lag. For example, a series in which only the most recent value is significant in predicting future values will have a large $k$ value (i.e., rapid decay in significance, or, equivalently, rapid increase of variance in dimension parallel to that lag). Conversely, time series in which previous lags are significant in predicting future values will have a smaller $k$ value.

\section{MLPs for Time Series Forecasting}

An MLP is a function of the following form:

$$
f\left(\mathbf{x}^{n}\right)=h(u) \text { where } u=\sum_{j=0}^{Q} w_{k j} g\left(\sum_{i=0}^{P} w_{j i} x_{i}^{n}\right)
$$

where $P$ is the number of inputs, $Q$ is the number of units in a hidden layer, $x_{i}^{n}$ is the input at unit $i$ from example $n, w_{j i}$ is a numerical weight connecting input unit $i$ with hidden unit $j$, and $w_{k j}$ is the weight connecting hidden unit $j$ with output unit $k$. The function $g(x)$ is either a sigmoid (i.e., $\left.g(x)=(1+\exp (-x))^{-1}\right)$ or some other continuous, differentiable, nonlinear function. For regression problems $h(u)$ is the identity function (i.e., $h(u)=u$ ), and for classification problems $h(u)$ is a sigmoid. 
Thus, an MLP with some given architecture and weight vector $\mathbf{w}$, provides a mapping from an input vector $\mathbf{x}$ to a predicted output $\mathrm{y}$ given by $\mathrm{y}=f(\mathbf{x}, \mathbf{w})$. For time series forecasting tasks performed in this paper, the vector $\mathbf{x}=\left(x_{1}, x_{2}, \ldots, x_{\mathrm{D}}\right)$ is assumed to be a vector of lagged returns in which $x_{1}=r_{t-1}, x_{2}=r_{t-2}$, etc. Given some data, $D$, consisting of $n$ independent items $\left(\mathbf{x}^{1}, \mathrm{y}^{1}\right), \ldots,\left(\mathbf{x}^{N}, \mathrm{y}^{N}\right)$, the objective is to find a suitable w.

\subsection{Maximum Likelihood Training}

The conventional approach to finding the weight vector $\mathbf{w}$ is to use a gradient descent method to find a weight vector that minimizes the error between the network output value, $f(\mathbf{x}, \mathbf{w})$, and the target value, $\mathrm{y}$. In the following we assume that the objective is to predict the direction of change in the next value of the return series; more specifically, the probability that the direction of change is positive. Therefore, under the assumption that the target values are binary $(y=1$ for a positive change; $y=0$ for a negative change), then the likelihood of observing the training data $D$, given some weight vector $\mathbf{w}$ is given by

$$
\begin{aligned}
p(D \mid \mathbf{w}) & =\prod_{n}\left(f\left(\mathbf{x}^{n}, \mathbf{w}\right)^{y^{n}}+\left(1-f\left(\mathbf{x}^{n}, \mathbf{w}\right)\right)^{\left(1-y^{n}\right)}\right) \\
& =\exp \sum_{n}\left(y^{n} \ln f\left(\mathbf{x}^{n}, \mathbf{w}\right)+\left(1-y^{n}\right) \ln \left(1-f\left(\mathbf{x}^{n}, \mathbf{w}\right)\right)\right)
\end{aligned}
$$

We wish also to specify a prior distribution for the weights, and we assume that this distribution is Gaussian with mean 0 and inverse variance $\alpha$. Thus,

$$
p(\mathbf{w})=\left(\frac{\alpha}{2 \pi}\right)^{1 / 2} \exp \left(-\frac{\alpha}{2} \sum_{i=1}^{m} w_{i}^{2}\right)
$$

We wish to find the weight vector which maximises the product of the likelihood and the prior. This can be shown to be equivalent to minimizing the following error term:

$$
E=-\sum_{n=1}^{N} y^{n} \ln f\left(\mathbf{x}^{n}, \mathbf{w}\right)+\left(1-y^{n}\right) \ln \left(1-f\left(\mathbf{x}^{n}, \mathbf{w}\right)\right)+\frac{\alpha}{2} \sum_{i=1}^{m} w_{i}^{2}
$$

The first term in this expression is commonly referred to as the 'cross-entropy error'; the second term is a 'regularization' term. Effectively, the regularization term punishes weight vectors in which the magnitude of weights is large, thereby helping reduce the possibility of overfitting to the training data. Note that aside from the weights, there is only one free parameter in the above equation: $\alpha$, which specifies the inverse variance for the weights prior.

\subsection{Lag-Dependent Regularization}

The regularization term in Equation 6 treats all weight equally. However, by separating weights into groupings, it is possible to implement a lag-dependent regularization, which achieves a similar result to the variance parameterization described in the case of generative models. Consider the use of non-spherical kernels of the form described in 
Section 2 above. From a discriminative classifier perspective, this is effectively making the assumption that noise in the training examples varies in such a way that input dimensions corresponding to recent returns are less noisy than inputs corresponding to less recent returns. We can build this assumption into an MLP by using separate regularization coefficients for different families of input-to-output layer weights. Specifically, weights fanning our from inputs corresponding to recent returns should have smaller weight-regularization coefficients than weights fanning out from inputs corresponding to less recent returns.

We first consider weights in the input-to-hidden layer weights. We use the notation $w_{i_{p} h_{q}}$ to represent the weight connecting input unit $i_{p}$ with hidden unit $h_{q}\left(i_{0}\right.$ represents the bias). Separating these weights into $D+1$ groups, where $D$ is the input dimensionality and $Q$ is the number of units in the hidden layer, we have

$$
\frac{\alpha}{2}\left(\sum_{j=1}^{Q} w_{i_{0} h_{j}}^{2}+\sum_{j=1}^{Q} w_{i_{1} h_{j}}^{2}+\sum_{j=1}^{Q} w_{i_{2} h_{j}}^{2}+\ldots+\sum_{j=1}^{Q} w_{i_{D}} h_{j}^{2}\right)
$$

We now apply exponential scaling factor of $e^{k(n-1)}$ to weights fanning from the input nodes 1 to $D$ giving

$$
\frac{\alpha}{2}\left(\sum_{j=1}^{Q}{w_{i} h_{j}}^{2}+e^{0 k} \sum_{j=1}^{Q}{w_{i} h_{j}}^{2}+e^{k} \sum_{j=1}^{Q} w_{i_{2} h_{j}}{ }^{2}+\ldots+e^{(D-1) k} \sum_{j=1}^{Q} w_{i_{D} h_{j}}{ }^{2}\right)
$$

which can be more succinctly expressed as

$$
\frac{\alpha}{2}\left(\sum_{j=1}^{Q} w_{i_{0} h_{j}}^{2}+\sum_{n=1}^{D}\left(e^{k(n-1)} \sum_{j=1}^{Q} w_{i_{n} h_{j}}^{2}\right)\right)
$$

The hidden-to-output layer weights can be treated as a single group. Incorporating these, the full regularization expression becomes

$$
\frac{\alpha}{2}\left(\sum_{j=1}^{Q} w_{i_{0} h_{j}}^{2}+\sum_{n=1}^{D}\left(e^{k(n-1)} \sum_{j=1}^{Q} w_{i_{n} h_{j}}^{2}\right)+\sum_{j=0}^{Q} w_{h_{j} o}{ }^{2}\right)
$$

Note that as with the generative approach described in Section 2, this modification still introduces two additional parameters: a parameter controlling the rate at which the value of the regularization coefficient varies with input lag $k$, and a parameter controlling the overall level of regularization, $\alpha$.

\section{Experiments}

We have applied the approach we have described to the daily close price of three financial time series: the Australian All Ordinaries (AORD) index, the Dow Jones Industrial Average (DJIA) index, and the Australian-U.S. Foreign Exchange (AUSE) rate. In each case we used a 20-year out-of-sample forecast period from 1 January 
1987 to 31 December 2006. Forecasts were performed in 25-day forecast windows, in which the model was constructed using the data points immediately preceding the forecast period. The number of data points used for model construction was 500 (approx. 2 years). This model was then used to predict the directional change probability for each data point in the forecast window. We note that the 25-day forecasting window period was chosen entirely for computational reasons, and there is no reason why a separate model cannot be constructed for a single prediction. The number of lags used in the input vector (i.e., the input dimensionality) was five. The number of hidden layer units was 50. As with the work described in [9], here, too, we are concerned with predicting the direction of change. That is, rather than forecasting the values of the return, we forecast the (probability of) the sign of the return.

\subsection{Testing Directional Forecast Accuracy}

An obvious measure of direction-of-change forecast accuracy is the fraction of days in some test period for which the sign is predicted correctly, and we refer to this as the sign ratio $(S R)$. We would like to know whether the value of $S R$ differs significantly from what we would expect if the signs of the actual and forecast predictions were independent. If $P$ is the fraction of days in the out-of-sample test period for which the actual movement is up, and $1 \hat{P}$ is the fraction of days for which the predicted movement is up, the expected fraction of days corresponding to a correct upward prediction is $P \times \hat{P}$, and the expected fraction of days corresponding to a correct downward prediction is $(1-P) \times(1-\hat{P})$. Thus, the expected fraction of correct predictions is $(P \times \hat{P})+((1-P) \times(1-\hat{P}))$. Since this is just the success ratio that we would expect if the signs of the actual and forecast predictions are independent, this is known as the sign independence ratio (SRI) [12]:

$$
S R I=(P \times \hat{P})+((1-P) \times(1-\hat{P})),
$$

Pesaran \& Timmermann (1992) show how SR and SRI can be combined to produce a directional accuracy test in which values for the test are normally distributed under the assumption that predicted and forecast values are independently distributed. The variance in $S R I$ and $S R$ can be calculated as follows:

$$
\begin{gathered}
\operatorname{var}(S R I)=\frac{1}{n}\left[(2 \hat{P}-1)^{2} P(1-P)+(2 P-1)^{2} \hat{P}(1-\hat{P})+\frac{4}{m} P \hat{P}(1-P)(1-\hat{P})\right] \\
\operatorname{var}(S R)=\frac{1}{n} S R I(1-S R I) .
\end{gathered}
$$

The value of the Pesaran-Timmermann test, which we refer to as the PT-score, is given by:

$$
\text { PT-score }=\frac{S R-S R I}{\sqrt{\operatorname{var}(S R)-\operatorname{var}(S R I)}} \sim N(0,1),
$$

Although the PT-score provides a measure of how statistically significant a set of forecasts is, it does not distinguish between models that differ in some important 
respects. For example, it is often useful to analyze results of classification tasks using a confusion matrix. For binary classification tasks, this will be a 2 by 2 matrix

$$
\begin{aligned}
& \text { Act }+ \text { Act - } \\
& \text { Pred }+\left[\begin{array}{ll}
T P & F P \\
F N & T N
\end{array}\right]
\end{aligned}
$$

where the rows represent predicted positives and predicted negatives, and the columns represent actual positives and actual negatives. The problem is that many different confusion matrices can result in the same PT-score. Thus, although the Pesaran-Timmermann test provides a measure of the statistical significance of a set of directional forecasts, it does not take into account the different nature of the models in respect to their ability to predicted positives/negatives.

One of the advantages of predicting the posterior probability of a directional change is that we can then use these probabilities to select the threshold for our classification. For example, we may only choose to make a directional forecast of up if the posterior probability exceeds a value of, say, 60\%. For this reason, it is important to have a measure of how our classifier performs not at just a $50 \%$ decision threshold, but across the whole range of thresholds. Receiver Operating Characteristic (ROC) curves provide such a measure. Plotting the true positive rate against the false positive rate for decision thresholds from 0 to 1 produces an ROC curve. The area under the curve provides a convenient single-value summary of the classifier's performance.

\subsection{Results}

Table 1 and 2 show the respectively the PT-score and area under ROC curve for the Australian All Ordinaries Index corresponding to a range of values for $\alpha$ and $k$ from Equation 10. The highest value in each table is shown in bold, and in both tables corresponds to $\alpha=1.50$ and $k=0.60$. Table 3 show the critical values for PT-score. Thus the observed PT-score of 5.135 is significant at the $10^{-6}$ level.

Table 1. PT-scores for Australian All Ordinaries (AORD) index

\begin{tabular}{ccccccccccc}
\hline $\mathrm{A}$ & \multicolumn{7}{c}{$k$} \\
\hline & 0.08 & 0.10 & 0.20 & 0.40 & 0.60 & 0.80 & 1.00 & 1.50 & 2.00 & 3.00 \\
\hline 0.000 & 1.472 & 1.472 & 1.472 & 1.472 & 1.472 & 1.472 & 1.472 & 1.472 & 1.472 & 1.472 \\
0.001 & 1.404 & 1.404 & 1.355 & 1.472 & 1.671 & 1.915 & 2.126 & 2.058 & 2.626 & 1.404 \\
0.010 & 2.099 & 2.118 & 2.141 & 2.212 & 2.813 & 2.896 & 2.856 & 3.174 & 1.570 & 2.099 \\
0.100 & 2.103 & 2.081 & 1.964 & 1.979 & 2.224 & 2.889 & 3.028 & 2.228 & 2.618 & 2.103 \\
0.500 & 2.832 & 2.332 & 3.039 & 1.916 & 3.523 & 2.607 & 2.194 & 2.776 & 3.284 & 2.832 \\
1.00 & 3.224 & 4.156 & 2.220 & 4.297 & 3.242 & 4.042 & 3.775 & 3.711 & 2.194 & 3.224 \\
1.50 & 4.003 & 3.438 & 2.649 & 3.550 & $\mathbf{5 . 1 3 5}$ & 4.087 & 4.110 & 2.814 & 2.469 & 4.003 \\
2.00 & 2.968 & 3.467 & 1.800 & 2.840 & 3.786 & 3.584 & 2.299 & 0.421 & 0.612 & 2.968 \\
10.0 & 1.349 & 1.890 & 2.719 & 2.468 & 1.436 & 0.387 & 0.928 & -1.266 & 1.496 & 1.349 \\
\hline
\end{tabular}


Table 2. Area under ROC curve for Australian All Ordinaries (AORD) index

\begin{tabular}{ccccccccccc}
\hline$\alpha$ & \multicolumn{10}{c}{$k$} \\
\hline & 0.08 & 0.10 & 0.20 & 0.40 & 0.60 & 0.80 & 1.00 & 1.50 & 2.00 & 3.00 \\
\hline 0.000 & 0.512 & 0.512 & 0.512 & 0.512 & 0.512 & 0.512 & 0.512 & 0.512 & 0.512 & 0.512 \\
0.001 & 0.511 & 0.511 & 0.510 & 0.512 & 0.512 & 0.513 & 0.517 & 0.521 & 0.523 & 0.511 \\
0.010 & 0.515 & 0.516 & 0.516 & 0.516 & 0.519 & 0.520 & 0.522 & 0.523 & 0.519 & 0.515 \\
0.100 & 0.516 & 0.514 & 0.515 & 0.518 & 0.520 & 0.526 & 0.524 & 0.521 & 0.523 & 0.516 \\
0.500 & 0.521 & 0.523 & 0.529 & 0.527 & 0.528 & 0.531 & 0.526 & 0.525 & 0.530 & 0.521 \\
1.00 & 0.533 & 0.534 & 0.530 & 0.537 & 0.528 & 0.537 & 0.536 & 0.532 & 0.520 & 0.533 \\
1.50 & 0.533 & 0.531 & 0.529 & 0.540 & $\mathbf{0 . 5 4 5}$ & 0.541 & 0.539 & 0.529 & 0.523 & 0.533 \\
2.00 & 0.529 & 0.533 & 0.526 & 0.532 & 0.540 & 0.540 & 0.529 & 0.502 & 0.510 & 0.529 \\
10.0 & 0.519 & 0.523 & 0.524 & 0.522 & 0.513 & 0.511 & 0.508 & 0.492 & 0.501 & 0.519 \\
\hline
\end{tabular}

Table 3. Critical values for PT-score

\begin{tabular}{ccccccccc}
\hline Sig. level & 0.05 & 0.01 & $10^{-3}$ & $10^{-4}$ & $10^{-5}$ & $10^{-6}$ & $10^{-7}$ & $10^{-8}$ \\
\hline PT-score & 1.645 & 2.327 & 3.090 & 3.719 & 4.265 & 4.753 & 5.199 & 5.612 \\
\hline
\end{tabular}

By observing the column corresponding to $k=0.6$, it can be seen from both tables that as $\alpha$ is increased the PT-score and Area steadily rise to a maximum value, and then begin to decline. This can be explained by the fact that a small value of $\alpha$ provides only a small penalty against large weights, thus leading to overfitting on the training data. Conversely, a large $\alpha$ imposes a high penalty on large weights, leading to underfitting on the training data. In both cases (i.e., overfitting and underfitting), we have suboptimal performance on holdout data.

Now consider the rows of each table. It can be seen that the maximum values in each row correspond to $k$ values in the vicinity of 0.4 to 0.8 . While the pattern observed when $k$ is increased from 0.08 to 3.00 is not one of steadily rising to a maximum, and then declining (i.e., the maximum is not as clearly pronounced as is the case for varying $\alpha$ ), the value of $k$ does clearly affect the performance on holdout data. In fact, it is interesting to compare these results with those obtained by using a fixed number of inputs without any differentiation on the prior distribution of weight families. Using 1, 2, 3, 4 and 5 inputs yields respectively the following values for the area under ROC curve: $0.534,0.523,0.525,0.534$ and 0.529 . These values are well below the value of 0.545 achieved using differentiated priors. It is interesting to note that the best values for the MLP approach $(\mathrm{PT}$-score $=5.135$, Area $=0.545)$ are very close to those for the density estimation-based approach described in Section 2 (PT-score = 5.704, Area $=0.543$ ).

We performed the same experiments on the Australian-U.S. Foreign Exchange rate and the Dow Jones Industrial Average index. For the exchange rate, the best values obtained for PT-score and Area under ROC curve were 2.72 and 0.517 , which are not as significant as the values of 3.22 and 0.521 obtained using the density estimationbased approach. Results for the DJIA were not statistically significant, either for the neural networks approach or the density estimation-based approach. 


\section{Conclusions}

A lag-dependent regularization technique has been proposed for use with MLPs applied to financial time series forecasting tasks. The technique is motivated by the intuitive notion that recent values of the series should have more influence than less recent values in predicting future values. The technique has been tested on three financial datasets, with directional forecast accuracy found to be significant on two of these datasets. A disadvantage of the MLP approach, as compared with a density estimation-based approach that we have previously proposed, is that in addition to the value of $\alpha$ and $k$, MLPs also depend on factors such as starting weights. We are currently applying the regularization technique described here within a Bayesian MLP learning framework. The integrative nature of the Bayesian framework is expected to reduce some of the variation between results obtained from different starting weights.

\section{References}

1. Cybenko, G.: Approximation by superpositions of a sigmoidal function. Mathematics of Control, Signals and Systems 2, 304-314 (1989)

2. Adya, M., Collopy, F.: How effective are neural networks at forecasting and prediction? a review and evaluation. Journal of Forecasting 17, 481-495 (1998)

3. Chatfield, C.: Positive or negative? International Journal of Forecasting 11, 501-502 (1995)

4. Tkacz, G.: Neural network forecasting of Canadian gdp growth. International Journal of Forecasting 17, 57-69 (2001)

5. Bishop, C.M.: Neural Networks for Pattern Recognition. Oxford University Press, Oxford (1995)

6. Akaike, H.: A new look at statistical model evaluation. IEEE Transactions on Automatic Control AC-19, 716-723 (1974)

7. Schwarz, G.: Estimating the dimension of a model. Annals of Statistics 6, 461-464 (1978)

8. Franses, P.H., van Dijk, D.: Non-Linear Time Series Models in Empirical Finance. Cambridge University Press, Cambridge (2000)

9. Skabar, A.: A kernel-based technique for direction-of-change financial time series forecasting. In: Bubak, M., van Albada, G.D., Dongarra, J., Sloot, P.M.A. (eds.) ICCS 2008, Part II. LNCS, vol. 5102, pp. 441-449. Springer, Heidelberg (2008)

10. Parzen, E.: On the estimation of a probability density function and mode. Annals of Mathematical Statistics 33, 1065-1076 (1962)

11. Duda, R.O., Hart, P.E.: Pattern Classification and Scene Analysis. John Wiley and Sons, New York (1974)

12. Pesaran, M.H., Timmermann, A.: A simple non-parametric test of predictive performance. Journal of Business \& Economic Statistics 10, 461-465 (1992) 